of wholemeal flour is said to be poorly absorbed because of the presence of large amounts of phytic acid. There have also been rumours that the added inon may likewise be poorly absorbed.

The barring of low-extraction flour from the diet and its replacement by wholemeal would therefore cause a slight increase in the iron intake, which might be counterbalanced by poor absorption. It seems improbable that this minor change in iron intake would have any noticeable effect on health. If the white flour banned from the diet were to be replaced by extra sugar or fat, however, the change in inon intake would be in the reverse direction and might be more serious.

\section{Treatment for Dandruf}

Q.-What is the present treatment for dandruff?

A.- That there are so many treatments for dandruff is testimony that none of them is completely effective or reliable. In what follows it should be understood that there is no permanent cure. With the treatments mentioned it is often possible to clear up the condition completely for the time, but relapse usually occurs in the course of some weeks after treatment has stopped.

In many cases the condition can be completely controlled with a shampoo alone. A $10 \%$ solution of cetrimide used as a shampoo once a week is sometimes completely successful. Other antiseptics, such as hexachlorophane, are also useful and form the effective ingredient of various proprietary preparations. Coal-tar derivatives are less effective. In recent years preparations such as " selsun" and "lenium" containing selenium sulphide have been found to be remarkably

\section{Notes and Comments}

Aphthous Ulcers.-Dr. W. H. CRICHTON (Polstead Heath, nr. Colchester) writes: With reference to the question on aphthous ulcers (“Any Questions ?" 28 December, p. 1644), a similar question was answered previously in the journal (3 September 1960 , p. 748) and a therapeutic trial of lozenges containing hydrocortisone hemisuccinate was advised. I have had occasion to prescribe these only once since I read this advice, and the result was magical in patient subject to recurrent manifestations of these ulcers. As no mention of this remedy is made in the current answer I should be grateful to know if hydrocortisone hemisuccinate is still effective. Used as a shampoo once a week or fortnight they are probably more effective than any other preparation. They have the disadvantage of being toxic materials, and it is said that they may encourage the hair to fall out, though I have not myself been convinced of this.

In a few cases the use of a shampoo does not seem to be surricient. When this is so a spirit lotion such as the following may be applied twice daily: Chloral hydrate, 2 parts ; salicylic acid, 3 parts ; castor oil, 4 parts; spirit to 100 .

recommended in the treatment of aphthous ulcers.

OUR EXPERT replies: The case that Dr. Crichton mentioned as having responded dramatically to hydrocortisone hemisuccinate illustrates the difficulties encountered in dealing with aphthous ulceration of the mouth. A good response to a variety of treatments has been recorded in a few cases, but unfortunately there is no remedy as yet which gives consistently good results in every case. Hydrocortisone hemisuccinate is certainly worth a trial, but in series of cases its efficacy seems to be no greater than that of the other remedies mentioned.

\title{
New Appliances
}

\section{Radio-Ryle's Tube}

Mr. R. MArcus, consultant surgeon, Clatterbridge Hospital ; Mr. L. E. MUSSEL, principal physicist, Clatterbridge Hospital; and Dr. J. WATT, lecturer, Department of Pathology, Liverpool University, write: The radio-Ryle's tube has been devised to provide a simple and accurate method of locating the position of the tip of a Ryle's tube in the stomach without the use of radiography. In

tronics workshop (the components costing only a few pounds). While full details of the physical principles and the electronic circuit are published elsewhere (Mussel, Marcus, and Watt, 1964), the essential features of its design, construction, and use are as follows.

The metal in the end of a No. 12 Frenchgauge plastic Ryle's tube is replaced by a

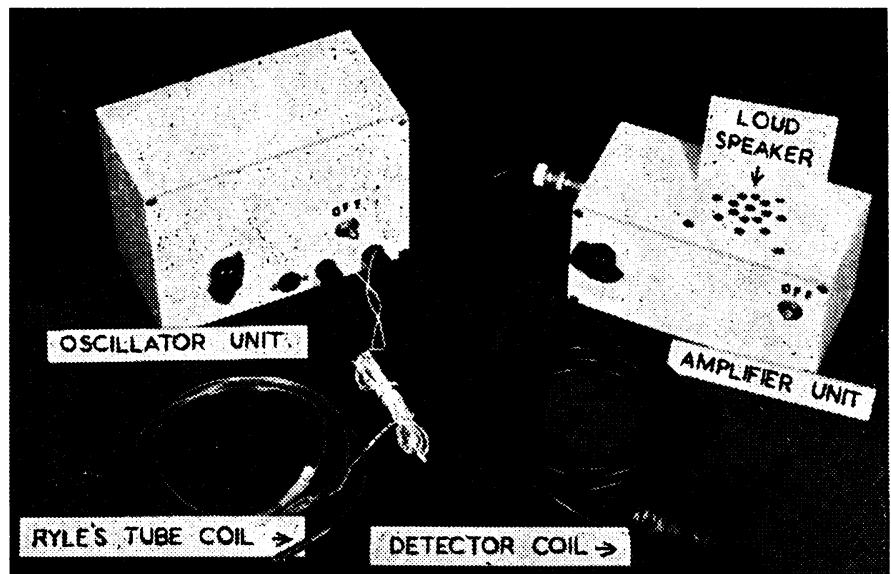

the augmented histamine test the tip of the tube can be positioned initially just to the left of the midline-that is, at the angulusand thereafter its position can be checked as often as required throughout the test.

The radio-Ryle's tube (see Fig.) can be simply and inexpensively built in an elec- small iron-cored coil about $\frac{5}{8}$ in. $(1.6 \mathrm{~cm}$.) long and $\frac{1}{8}$ in. $(0.3 \mathrm{~cm}$.) diameter. This coil is connected to fine wires led back along the Ryle's tube and then joined, by a stouter lead, to an oscillator unit supplying a signal of $30 \mathrm{~mW}$ maximum power at $2 \mathrm{kc}$./s. The radiated signal is picked up by a larger detector coil (wound from $2 \mathrm{oz}$. (57 g.) of 36 swg wire on a laminated iron core some 2 in. ( $5 \mathrm{~cm}$.) long), and this coil is connected by a screened lead to an audio-frequency amplifier feeding a small loudspeaker. All the electronic circuits are transistorized, so that they are completely portable and supplied from 9-volt batteries.

In use the detector coil is moved about near the intubated patient, and when the maximum volume of sound is obtained in the loudspeaker then it is known that the detector coil is at its closest distance from the Ryle's tube coil, whose position is thus found. In practice a set of rules is used which allows the sharp dip in the polar diagram of the detector coil (along its axis) to provide a more precise indication of the position of the oscillator coil. Under these conditions a positional accuracy of about $1 \mathrm{~cm}$. can be expected when the detector coil is 7-10 cm. from the end of the Ryle's tube (with the accuracy only falling off slowly as the distance between the coils is increased).

During passage of the tube the signals may be located by scanning with the detector coil from either the front or the back of the patient, the former being more convenient. The set of rules provided allows the direction as well as position of the Ryle's tube coil to be determined.

The incorporation of the small coil in the end of the Ryle's tube is readily achieved without increasing the bulk of the tip. In consequence, passage of the radio-Ryle's tube is no more difficult than that of an ordinary Ryle's tube. The aspiration of the gastric juices is in no way impeded by the fine wires in the lumen.

\section{RBFERENCB}

Mussell, L. E., Marcus, R., and Watt, J. (1964). Phys. in Med. Biol., 9, 73. 